\title{
On Minkowskian Branching Structures
}

\author{
Leszek Wroński and Tomasz Placek*
}

November 4, 2018

\begin{abstract}
Contrary to its initial idea, Belnap's (1992) theory of Branching Space-Times (BST) has models in which histories do not resemble relativistic space-times or any other physical space-times. The aim of this paper is to define a certain class of BST models, called "Minkowskian Branching Structures" (MBS), in which histories are isomorphic to Minkowski space-time. By focusing on these models rather than on general BST models, we hope that one may be able to improve on earlier BST analyzes of physical phenomena. Also, introducing MBS' sets the stage for recent discussions about whether or not 'branching is a bad idea', physically speaking.
\end{abstract}

\section{Introduction}

Branching Space-Times (BST) of Belnap (1992) is a possible-worlds theory that intends to combine, in a rigorous fashion, indeterminism, relativity and modalities. Yet, ironically, BST allows for histories that resemble neither relativistic space-times nor any other physical space-times. Müller, Belnap and Kishida (2006) discovered a structure called $M_{2}$ which, while being a BST model, is in no way similar to the intended pictures of the theory. Our aim in this paper is thus to define a certain class of BST models called "Minkowskian Branching Structures" (MBS), in which histories are isomorphic to

${ }^{*}$ We read earlier versions of this paper at the seminar 'Chaos and Quantum Information' held at the Jagiellonian University in Kraków on April 16, 2007 and at the seminar 'On Determinism' held at the University of Bonn on April 20, 2007. For comments and valuable discussions we are grateful to the audiences, and in particular, to Dr. Thomas Müller. Authors' address: Department of Philosophy, Jagiellonian University, Grodzka 52, 31-044 Kraków, Poland; LW's email: elwro1@gmail.com and TP's email: uzplacek@cyf-kr.edu.pl. 
Minkowski space-time. In defining the notion we follow the lead of Müller (2002), yet with two crucial diversions. First, we remove Müller's finiteness assumptions, as they prohibit the introduction of 'interesting' infinite structures. Second, we improve on Müller's failed proof of the most desired feature of MBS, namely that every history is isomorphic to Minkowski space-time. To this end we assume a certain topological postulate.

By concentrating on MBS' rather than general BST models, one may avoid a certain awkwardness inherent in earlier analyses of some physical phenomena, done from a BST perspective 1 In essence, they tried to fit information about the location of objects into models with no metric features. This problem will not arise in our structures.

Singling out the class of MBS models has yet another advantage: It has been alleged in the philosophy of physics community that branching is a bad idea 2 Yet, to sensibly discuss the pros and cons of branching, as applied to physics, one had better have a class of BST models which have some pretense at being 'physically realistic'.

The paper is organized as follows. In Section 2 we review some definitions and theorems of BST which we will need later. Section 3 defines and discusses Minkowskian Branching Structures. The final section 4 states our conclusions and an open problem.

\section{Branching Space-Times}

The theory of Branching Space-Times (BST) of Belnap (1992), combines objective indeterminism and relativity in a rigorous way. Its primitives are a nonempty set $W$ (called "Our World", interpreted as the set of all possible point events) and a partial ordering $\leqslant$ on $W$, interpreted as a "causal order" between point events.

There are no "Possible Worlds" in this theory; there is only one world, Our World, containing all that is (timelessly) possible. Instead, a notion of "history" is used, as defined below:

\footnotetext{
${ }^{1}$ To give some examples, the Bell / Aspect Theorems were analyzed in Belnap and Szabó (1996), Kowalski and Placek (1999), and Müller and Placek (2001). A branching perspective on quantum holism was put forward in Placek (2004). A BST reading of the consistent histories interpretation of quantum mechanics was given in Müller (2007). Some questions from the philosophy of time were discussed, from a BST perspective, in Müller (2006).

${ }^{2}$ See for instance J. Earman's talk at the Ontology of Space-Time conference, Montreal 2006.
} 
Definition $1 A$ set $h \subseteq W$ is upward-directed iff $\forall e_{1}, e_{2} \in h \exists e \in h$ such that $e_{1} \leqslant e$ and $e_{2} \leqslant e$.

$A$ set $h$ is maximal with respect to the above property iff $\forall g \in W$ such that $g \nsupseteq h, g$ is not upward-directed.

$A$ subset $h$ of $W$ is a history iff it is a maximal upward-directed set.

For histories $h_{1}$ and $h_{2}$, any maximal element in $h_{1} \cap h_{2}$ is called a choice point for $h_{1}$ and $h_{2}$.

A very important feature of BST is that histories are closed downward: if $e_{1} \leqslant e_{2}$ and $e_{1} \notin h$, then $e_{2} \notin h$. In other words, there is no backward branching among histories in BST. No two incompatible events are in the past of any event; equivalently: the past of any event is "fixed", containing only compatible events.

We will now give the definition of a BST model; for more information about BST in general see Belnap (1992).

Definition $2\langle W, \leqslant\rangle$ where $W$ is a nonempty set and $\leqslant$ is a partial ordering on $W$ is a model of $B S T$ if and only if it meets the following requirements:

1. The ordering $\leqslant$ is dense.

2. S has no maximal elements.

3. Every lower bounded chain in $W$ has an infimum in $W$.

4. Every upper bounded chain in $W$ has a supremum in every history that contains it.

5. (Prior choice principle ('PCP')) For any lower bounded chain $O \in$ $h_{1}-h_{2}$ there exists a point $e \in W$ such that $e$ is maximal in $h_{1} \cap h_{2}$ and $\forall e^{\prime} \in O e<e^{\prime}$.

Note that BST has the causal ordering $\leqslant$, yet in general it has neither space-time points nor a space-time (i.e., a collection of space-time points). The intuition behind space-time points is that we want to be able to say that something happens "in the same space-time point" in different histories. A triple $\langle W, \leqslant, s\rangle$ is a "BST model with space-time points" $(\mathrm{BST}+\mathrm{S})$ iff $\langle W, \leqslant\rangle$ is a BST model and $s$ (from the expression "space-time point") is an equivalence relation on $W$ such that 1 ) for each history $h$ in $W$ and for each equivalence class $s(x), x \in W$, the intersection $h \cap s(x)$ contains exactly one element and 2) $s$ respects the ordering: for equivalence classes $s(x), s(y)$ and histories $h_{1}, h_{2}, s(x) \cap h_{1}=s(y) \cap h_{1}$ iff $s(x) \cap h_{2}=s(y) \cap h_{2}$, and the same for " $<$ " and ">". As Müller (2005) shows, not every BST model 
can be extended to a BST $+\mathrm{S}$ model. Moreover, there are BST $+\mathrm{S}$ models, whose space-time $S$ has nothing in common with any physically motivated space-time - the structure $M_{2}$ of Müller, Belnap and Kishida (2006) is an example.

\section{Introducing Minkowskian Branching Struc- tures}

We are now going to single out a class of BST models in which histories occur in Minkowski space-time. This part of our work is based on Müller's (2002) theory.

The points of Minkowski space-time are elements of $\mathbb{R}^{4}$, e.g. $x=\left\langle x^{0}, x^{1}, x^{2}, x^{3}\right\rangle$, where the first element of the quadruple is the time coordinate. The Minkowski space-time distance is a function $D_{M}^{2}: \mathbb{R}^{4} \times \mathbb{R}^{4} \rightarrow \mathbb{R}$ defined as follows (for $\left.x, y \in \mathbb{R}^{4}\right)$ :

$$
D_{M}^{2}(x, y):=-\left(x^{0}-y^{0}\right)^{2}+\sum_{i=1}^{3}\left(x^{i}-y^{i}\right)^{2}
$$

The natural ordering on the Minkowski space-time, call it "Minkowskian ordering $\leqslant_{M}$ ", is defined as follows $\left(x, y \in \mathbb{R}^{4}\right)$ :

$$
x \leqslant_{M} y \text { iff } D_{M}^{2}(x, y) \leqslant 0 \text { and } x^{0} \leqslant y^{0}
$$

We will say that two points $x, y \in \mathbb{R}^{4}$ are space-like related ("SLR" for short) iff neither $x \leqslant_{M} y$ nor $y \leqslant_{M} x$. Naturally, $x<_{M} y$ iff $x \neq y$ and $x \leqslant_{M} y$.

Now we need to provide a framework for "different ways in which things can happen" and for filling the space-times with content. For the first task we will need a set $\Sigma$ of labels $\sigma, \eta, \ldots$. (In contrast to Müller (2002), we allow for any cardinality of $\Sigma$ ). For the second task, we will use a so-called "state" function $S: \Sigma \times \mathbb{R}^{4} \rightarrow P$, where $P$ is a set of point properties (on this we just quote Müller saying "finding out what the right $P$ is is a question of physics, not one of conceptual analysis").

One could ask about the reasons for an extra notion of a "scenario". Why don't we build histories just out of points from $\mathbb{R}^{4} \times P$ ? The reason is that a member of BST's Our World has a fixed past. If two different trains of events lead to exactly the same event $E \in \mathbb{R}^{4} \times P$, the situation gives rise to two different point events, two different members of $W$. In contrast: for a point $\left\langle x, p_{0}\right\rangle$ from $\mathbb{R}^{4} \times P$ there can exist two different points $\left\langle y, p_{1}\right\rangle$ and 
$\left\langle y, p_{2}\right\rangle$ from $\mathbb{R}^{4} \times P$ such that $y<_{M} x$. This would be a case of backward branching, so the set $\mathbb{R}^{4} \times P$ is not a good candidate for the master set $W$ of any BST model.

The idea behind the concept of scenario is that every scenario corresponds to an $\mathbb{R}^{4}$ space filled with content 3 , where the content derives from the elements of $P$. Assuming that a certain state function $S$ is given, for any $\sigma, \eta \in \Sigma$ the set $C_{\sigma \eta} \subset \mathbb{R}^{4}$ is the set of "splitting points" between scenarios $\sigma$ and $\eta$; intuitively it is the set of points in which a choice between the two scenarios is made. All members of $C_{\sigma \eta}$ have to be space-like related. Of course a choice between $\sigma$ and $\eta$ is a choice between $\eta$ and $\sigma$, so $C_{\sigma \eta}=C_{\eta \sigma}$. The BST axiom of prior choice principle motivates our postulate that any two different scenarios split. Formally: $\forall \sigma, \eta \in \Sigma\left(\sigma \neq \eta \Rightarrow C_{\sigma \eta} \neq \emptyset\right)$.

The next requirement concerns triples of scenarios. Any set $C_{\sigma \eta}$ determines a region in which both scenarios coincide: namely, that part of $\mathbb{R}^{4}$ that is not in the Minkowskian sense strictly above any point from $C_{\sigma \eta}$. Following Müller we call it the region of overlap $R_{\sigma \eta}$ between scenarios $\sigma, \eta$ defined as below:

$$
R_{\sigma \eta}:=\left\{x \in \mathbb{R}^{4} \mid \neg \exists y \in C_{\sigma \eta} y<_{M} x\right\}
$$

(Of course it follows that for any $\sigma, \eta \in \Sigma C_{\sigma \eta} \subseteq R_{\sigma \eta}$.) Assuming the sets $C_{\sigma \eta}$ and $C_{\eta \gamma}$ are given, we get two regions of overlaps $R_{\sigma \eta}$ and $R_{\eta \gamma}$. At the points in the intersection of those two regions $\sigma$ coincides with $\eta$ and $\eta$ coincides with $\gamma$, therefore by transitivity $\sigma$ coincides with $\gamma$. In general we can say that for any $\sigma, \eta, \gamma \in \mathbb{R}^{4}$

$$
R_{\sigma \gamma} \supseteq R_{\sigma \eta} \cap R_{\eta \gamma}
$$

which, translated to a requirement on sets of splitting points, is

$$
\forall x \in C_{\sigma \gamma} \exists y \in C_{\sigma \eta} \cup C_{\eta \gamma} y \leqslant_{M} x .
$$

In his paper Müller put another requirement on $C_{\sigma \eta}$ : finitude. The motivation was to exclude splitting along a "simultaneity slice". The strong requirement of finitude, however, excludes many more types of situations in which splitting is not continuous or happens in a region of space-time of finite diameter. In the present paper we drop this requirement, not putting any restrictions on the cardinality of $C_{\sigma \eta}$ for any $\sigma, \eta \in \Sigma$. As a side-note, this leads to the fact that in some models there may be choice points which are not intuitively connected with any splitting point. For details, see 5 in the Appendix.

\footnotetext{
${ }^{3}$ Fix a scenario $\alpha$. The above mentioned corresponding space filled with content is $A \subseteq \mathbb{R}^{4} \times P$ such that $\langle x, p\rangle \in A$ iff $S(\alpha, x)=p$.
} 
The state function assigns to each pair $\left\langle\right.$ a label from $\Sigma$, a point from $\left.\mathbb{R}^{4}\right\rangle$ an element of $P$. Colloquially, the state function tells us what happens at a certain point of the space-time in a given scenario 4

After Müller (2002), we now proceed to construct the elements of the MBS version of Our World; they will be equivalence classes of a certain relation $\leqslant_{S}$ on $\Sigma \times \mathbb{R}^{4}$. For convenience, we write the elements of $\Sigma \times \mathbb{R}^{4}$ as $x_{\sigma}$, where $x \in \mathbb{R}^{4}, \sigma \in \Sigma$. The idea is to "glue together" points in regions of overlap; hence the relation is defined as below:

$$
x_{\sigma} \equiv_{S} y_{\eta} \text { iff } x=y \text { and } x \in R_{\sigma \eta} .
$$

Müller provides a simple proof of the fact that $\equiv_{S}$ is an equivalence relation on $\Sigma \times \mathbb{R}^{4}$; therefore, we can produce a quotient structure. The result is the set $B$, which is the MBS version of Our World:

$$
B:=\left(\Sigma \times \mathbb{R}^{4}\right) / \equiv_{S}=\left\{\left[x_{\sigma}\right] \mid \sigma \in \Sigma, x \in \mathbb{R}^{4}\right\},
$$

where $\left[x_{\sigma}\right]$ is the equivalence class of $x$ with respect to the relation $\equiv_{S}$ :

$$
\left[x_{\sigma}\right]=\left\{x_{\eta} \mid x_{\sigma} \equiv_{S} x_{\eta}\right\} \text {. }
$$

Next, we define a relation $\leqslant_{S}$ on $B$ :

$$
\left[x_{\sigma}\right] \leqslant_{S}\left[y_{\eta}\right] \text { iff } x \leqslant_{M} y \text { and } x_{\sigma} \equiv_{S} x_{\eta},
$$

which (as Müller shows) is a partial ordering on $B$.

The goal would now be to prove that $\left\langle B, \leqslant_{S}\right\rangle$ is a model of BST. To do so, and in particular to prove the prior choice principle and requirement no. 4 from definition 2, we need to know more about the shape of the histories in MBS - that they are the intended ones.

\subsection{The shape of MBS histories}

We would like histories, that is, maximal upward-directed sets, to be sets of equivalence classes $\left[x_{\sigma}\right]$ (with respect to $\equiv_{S}$ ) for $x \in \mathbb{R}^{4}$ for some $\sigma \in \Sigma$. In other words, we wish to unambiguously refer to any history by a label from $\Sigma$, requiring one-to-one correspondence of the sets of histories and labels. This is Müller's (2002) Lemma 3 and our

Theorem 3 Every history in a given $M B S$ is of the form $h=\left\{\left[x_{\sigma}\right] \mid x \in \mathbb{R}^{4}\right\}$ for some $\sigma \in \Sigma$.

\footnotetext{
${ }^{4}$ We can look at the situation from a slightly different perspective: every label $\sigma$ is assigned a mapping $S_{\sigma}$ from $\mathbb{R}^{4}$ to $P$; see also the previous footnote.
} 
The problem is that, aside from minor brushing up required by the proof of the "right" direction, the proof of the "left" direction supplied in Müller (2002) needs to be fixed, as it does not provide adequate reasons for nonemptiness of an essential intersection $\bigcap \Sigma_{h}\left(z_{i}\right)$. More on that below. Let us divide the above theorem into two lemmas (4) and 8 ), corresponding to the directions and prove the "right" direction first. Until we prove the theorem we refrain from using the term "history" and substitute it with a "maximal upward-directed set" for clarity.

Lemma 4 If $h=\left\{\left[x_{\sigma}\right] \mid x \in \mathbb{R}^{4}\right\}$ for some $\sigma \in \Sigma$, then $h$ is a maximal upward-directed subset of $B$.

Proof: Let us consider $e_{1}, e_{2} \in h, e_{1}=\left[x_{\sigma}\right], e_{2}=\left[y_{\sigma}\right]$. Since $x, y \in \mathbb{R}^{4}$, there exists a $z \in \mathbb{R}^{4}$ such that $x \leqslant_{M} z$ and $y \leqslant_{M} z$. Therefore, $\left[x_{\sigma}\right] \leqslant_{S}\left[z_{\sigma}\right]$ and $\left[y_{\sigma}\right] \leqslant_{S}\left[z_{\sigma}\right]$, and so $h$ is upward-directed.

For maximality, consider a $g \subseteq B, g \nsupseteq h$ and assume $g$ is upward-directed. It follows that there exists a point $\left[x_{\eta}\right] \in g-h$ such that $\left[x_{\eta}\right] \neq\left[x_{\sigma}\right] \in h$. Since both points belong to $g$, which is upward-directed, there exists $\left[z_{\alpha}\right] \in g$ (note that we are not allowed to choose $\sigma$ as the index at that point) such that $\left[x_{\eta}\right] \leqslant_{S}\left[z_{\alpha}\right]$ and $\left[x_{\sigma}\right] \leqslant_{S}\left[z_{\alpha}\right]$. Therefore, $x_{\eta} \equiv_{S} x_{\alpha} \equiv_{S} x_{\sigma}$, and so we arrive at a contradiction by concluding that $\left[x_{\eta}\right]=\left[x_{\sigma}\right]$. Q.E.D.

The proof of the other direction is more complex and, what might be surprising, involves a topological postulate. First, we will need a simple definition:

Definition 5 For a given maximal upward-directed set $h$ and a point $x \in \mathbb{R}^{4}$, $\Sigma_{h}(x):=\left\{\sigma \in \Sigma \mid\left[x_{\sigma}\right] \in h\right\}$.

Consider now a given maximal upward-directed set $h \subseteq B$. With every lower bounded chain $L \subset \mathbb{R}^{4}$ we would like to associate a topology (called a "chain topology") on the set of $\Sigma_{h}(\inf (L))$. We define the topology by describing the whole family of closed sets, which is equal to $\left\{\emptyset, \Sigma_{h}(\inf (L))\right\} \cup$ $\left\{\Sigma_{h}(l) \mid l \in L\right\} \cup\left\{\cap\left\{\Sigma_{h}(l) \mid l \in L\right\}\right\}$. (Because $L$ is a chain, it is evident that the family is closed with respect to intersection and finite union). The postulate runs as follows:

Postulate 6 For every maximal upward-directed set $h \subseteq B$ and for every lower bounded chain $L \subset \mathbb{R}^{4}$ the "chain topology" described above is compact.

It is easily verifiable that in such a topology $\left\{\Sigma_{h}(l) \mid l \in L\right\}$ is a centred family of closed sets (every finite subset of it has a nonempty intersection). It is provable that the above postulate is equivalent to the following corollary: 
Corollary 7 For every maximal upward-directed set $h \subseteq B$ and for every chain $L \subset \mathbb{R}^{4}, \bigcap\left\{\Sigma_{h}(l) \mid l \in L\right\} \neq \emptyset$.

Lemma 8 If $h$ is a maximal upward-directed subset of $B$, then $h=\left\{\left[x_{\sigma}\right] \mid x \in\right.$ $\left.\mathbb{R}^{4}\right\}$ for some $\sigma \in \Sigma$.

The structure of the proof mimics Müller's (2002) proof. It is divided into three parts, the first and the last being reproduced here. On the other hand, the second part contains an error (as stated above, the statement that $\bigcap \Sigma_{h}\left(z_{i}\right) \neq \emptyset$ is not properly justified) and relies on the assumption that for every history $h$ and point $x \in \mathbb{R}^{4}$ the set $\Sigma_{h}(x)$ is at most countably infinite. We wish both to drop this assumption and correct the proof using the above topological postulate.

Proof: Suppose that $h$ is a maximal upward-directed subset of $B$. In order to prove the lemma, we will prove the following three steps:

1. If for some $\sigma, \eta \in \Sigma$ both $\left[x_{\sigma}\right] \in h$ and $\left[x_{\eta}\right] \in h$, then $x_{\sigma} \equiv_{S} x_{\eta}$.

2. There is a $\sigma \in \Sigma$ such that for every $\eta$, if $\left[x_{\eta}\right] \in h$, then $x_{\eta} \equiv_{S} x_{\sigma}$.

3. With the $\sigma$ from step $2, h=\left\{\left[x_{\sigma}\right] \mid x \in \mathbb{R}^{4}\right\}$.

Ad. 1. Since $h$ is maximal by assumption, there exists a $\left[y_{\gamma}\right] \in h$ such that $\left[x_{\sigma}\right] \leqslant_{S}\left[y_{\gamma}\right]$ and $\left[x_{\eta}\right] \leqslant_{S}\left[y_{\gamma}\right]$. These last two facts imply that $x_{\sigma} \equiv_{S} x_{\gamma} \equiv_{S} x_{\eta}$, so by transitivity of $\equiv_{S}$ we get $x_{\sigma} \equiv_{S} x_{\eta}$.

Ad. 2. Assume the contrary: $\forall \sigma \in \Sigma \exists\left[x_{\eta}\right] \in h, x_{\eta} \not_{S} x_{\sigma}$.

Take a point $\left[y_{\kappa}\right] \in h$. Accordingly, $\Sigma_{h}(y) \neq \emptyset$.

For each scenario $\sigma_{\alpha} \in \Sigma_{h}(y)$ we define a set $\Theta_{\alpha}=\left\{x \in \mathbb{R}^{4} \mid \exists \eta \in\right.$ $\left.\Sigma_{h}(y):\left[x_{\eta}\right] \in h \wedge x_{\sigma_{\alpha}} \not \equiv_{S} x_{\eta}\right\}$, which by our assumption is never empty. Colloquially, it is a set of the points that make the scenario a wrong candidate for the proper scenario from our lemma - the scenario "doesn't fit" the history at those points. For each scenario $\sigma_{\alpha}$ we would like to choose a single element of $\Theta_{\alpha}$, and to that end we employ a choice function $T$ defined on the set of subsets of $\mathbb{R}^{4}$ such that $T\left(\Theta_{\alpha}\right) \in \Theta_{\alpha}$, naming the element chosen by it as follows: $T\left(\Theta_{\alpha}\right):=x_{\alpha} \cdot \frac{5}{5}$

Observe that we will arrive at a contradiction if we prove that

$$
\bigcap_{\sigma_{\alpha} \in \Sigma_{h}(y)} \Sigma_{h}\left(x_{\alpha}\right) \neq \emptyset
$$

(since for any $\sigma_{\beta} \in \Sigma_{h}(y) \sigma_{\beta} \notin \Sigma_{h}\left(x_{\beta}\right)$ ). In order to apply our topological postulate, we will construct a chain $L=\left\{z_{0}, z_{1}, \ldots, z_{\omega}, \ldots\right\}$ of points in $\mathbb{R}^{4}$.

\footnotetext{
${ }^{5}$ Bear in mind that since $\alpha$ is a number serving just as an index for scenarios, $x_{\alpha}$ (like $x_{\beta}$ in the line below inequality 10 is a point from $\mathbb{R}^{4}$ and does not denote a point - scenario pair.
} 
It will be lower bounded by its initial element $z_{0}$. Moreover, we want it to be vertical, since in this way it will (if it does not have an upper bound itself) contain an upper bound of any point in $\mathbb{R}^{4}$, which will be needed in our proof.

We first define a function " $u p$ " which, given two points $a, b \in \mathbb{R}^{4}$, will produce a point $c \in \mathbb{R}^{4}$ such that $c$ has the same spatial coordinates as $a$ but is above $b$. In other words, if $a=\left\langle a^{0}, a^{1}, a^{2}, a^{3}\right\rangle \in \mathbb{R}^{4}, b=\left\langle b^{0}, b^{1}, b^{2}, b^{3}\right\rangle \in \mathbb{R}^{4}$, $u p(a, b):=\left\langle a^{0}+\left(\sum_{1}^{3}\left(a^{i}-b^{i}\right)^{2}\right)^{1 / 2}, a^{1}, a^{2}, a^{3}\right\rangle \in \mathbb{R}^{4}$. Notice that up is not commutative.

We proceed to define the above mentioned chain $L$ in the following way:

1. $z_{0}=u p\left(y, x_{0}\right)$.

$z_{1}=u p\left(z_{0}, x_{1}\right)$.

Generally, $z_{k+1}=u p\left(z_{k}, x_{k+1}\right)$.

2. Suppose $\rho$ is a limit number. Define $A_{\rho}:=\left\{z_{\beta} \mid \beta<\rho\right\} 6$. As you can see, $A_{\rho}$ is the part of our chain we have managed to construct so far. We need to distinguish two cases:

a) $A_{\rho}$ is upper bounded with respect to $\leqslant_{M}$. Then it has to have "vertical" upper bounds $t_{0}, t_{1} \ldots$ with spatial coordinates $t_{n}^{i}=z_{0}^{i}(i=1,2,3)$. In this case, we employ the above defined function $T$ to choose one of the upper bounds of $A_{\rho}$ :

$$
t_{\rho}:=T\left(\left\{t \in \mathbb{R}^{4} \mid \forall \beta<\rho z_{\beta} \leqslant_{M} t \wedge t^{i}=z_{0}^{i}(i=1,2,3)\right\}\right) .
$$

Then we put $z_{\rho}:=u p\left(t_{\rho}, x_{\rho}\right)$, arriving at the next element of our chain $L$.

b) if $A_{\rho}$ is not upper bounded with respect to $\leqslant_{M}$, then no matter which point in $\mathbb{R}^{4}$ we choose, it is possible to find a point from $A_{\rho}$ above it (since $A_{\rho}$ is vertical). Therefore, the set

$$
B_{\rho}=\left\{t \in A_{\rho} \mid x_{\rho} \leqslant{ }_{M} t\right\}
$$

is not empty. We put $\left[z_{\rho}\right]:=T\left(B_{\rho}\right)$, arriving at the next element of our chain $L$.

Notice that in our chain it might happen that while $\alpha<\beta, z_{\beta} \leqslant{ }_{M} z_{\alpha}, z_{0}$ is a lower bound of $L$. Therefore, our postulate 6 applies. By employing it and corollary 7 we infer that

$$
\bigcap_{\sigma_{\alpha} \in \Sigma_{h}(y)}\left\{\Sigma_{h}\left(z_{\alpha}\right) \mid z_{\alpha} \in L\right\} \neq \emptyset
$$

By our construction of the chain $L$, for all $\alpha$ it is true that $x_{\alpha} \leqslant_{M} z_{\alpha}$. Therefore, $\Sigma_{h}\left(z_{\alpha}\right) \subseteq \Sigma_{h}\left(x_{\alpha}\right)$. Thus, from 13 we immediately get

$$
\bigcap_{\sigma_{\alpha} \in \Sigma_{h}(y)} \Sigma_{h}\left(x_{\alpha}\right) \neq \emptyset
$$

\footnotetext{
${ }^{6}$ Again, $\beta$ is just an index, not a scenario, so $A_{\rho}$ is a subset of $\mathbb{R}^{4}$.
} 
which is the equation 10 that we tried to show. Therefore, we arrive at a contradiction and part 2 of the proof is complete.

Ad. 3. We have shown that there is a scenario $\sigma \in \Sigma$ such that all members of $h$ can be identified as $\left[x_{\sigma}\right]$ for some $x \in \mathbb{R}^{4}$. What remains is to show that the history cannot "exclude" some regions of $\{\sigma\} \times \mathbb{R}^{4}$, that is, to prove that for all $x \in \mathbb{R}^{4},\left[x_{\sigma}\right] \in h$. But in lemma 4 we have shown that $\left\{\left[x_{\sigma}\right] \mid x \in \mathbb{R}^{4}\right\}$ is a maximal upward-directed subset of $B$, so any proper subset of it cannot be maximal upward-directed. Q.E.D.

By showing lemmas 4 and 8 we have proved theorem 3 .

\subsection{The importance of the topological postulate}

So far it might seem that our topological postulate 6 is just a handy trick for proving lemma 8. To show its importance we will now prove that its falsity leads to the falsity of the lemma, and then present an example of a structure in which the lemma does not hold.

Theorem 9 If postulate 6 is false, then lemma 8 is also false.

Proof: Assume that our topological postulate does not hold. Therefore, there exists a maximal upward-directed set $h \subseteq B$ and a lower bounded chain $L \subset \mathbb{R}^{4}$ such that the chain topology is not compact. By rules of topology this is equivalent to the fact that there is a centred family of closed sets with an empty intersection. But all closed sets in the chain topology form a chain with respect to inclusion. Of course, if a part of a chain has an empty intersection, a superset of the part also has an empty intersection. We infer that

$$
\bigcap_{x \in L} \Sigma_{h}(x)=\emptyset
$$

from which, by definition 5, we get that

$$
\neg \exists \sigma \in \Sigma: \forall x \in L\left[x_{\sigma}\right] \in h,
$$

so there is no scenario $\sigma$ such that $h=\left\{\left[x_{\sigma}\right] \mid x \in \mathbb{R}^{4}\right\}$. Thus, lemma 8 is false. Q.E.D.

In the next subsection we show a situation in which lemma 8 does not hold. The construction resembles the $M_{1}$ structure of Müller, Belnap, and Kishida (2006). 


\subsection{When the topological postulate is false}

We will now show a situation in which lemma 8 does not hold. By fixing two spatial dimensions we will restrict ourselves to $\mathbb{R}^{2}$, the first coordinate representing time.

As usual, $\Sigma$ is the set of all scenarios of a world $B$. Let $C$ be the set of all splitting points:

$$
C:=\bigcup_{\sigma, \eta \in \Sigma} C_{\sigma \eta}
$$

We put

$$
C:=\{\langle 0, n\rangle \mid n \in \mathbb{N} \cup\{0\}\}
$$

The idea is that all splitting points are binary: any scenario passing through a given splitting point can go either "left" or "right". Since there are as many splitting points as natural numbers, we can identify $\Sigma$ with a set of 01-sequences. Another requirement on $\Sigma$ is that it contain only sequences with finitely many 0 s. Let $G$ be a subset of $\Sigma$ containing only the sequence without any $0 \mathrm{~s}$ and all sequences that have all their $0 \mathrm{~s}$ at the beginning. The elements of $G$ will be labeled as below:

$$
\begin{gathered}
\sigma_{0}=1111 \ldots \\
\sigma_{1}=01111 \ldots \\
\sigma_{2}=00111 \ldots \\
\sigma_{3}=00011 \ldots
\end{gathered}
$$

Let us next consider a sequence $Z_{i}^{M}$ of points in $\mathbb{R}^{2}$ such that for all $i \in$ $\mathbb{N} z_{i}=\langle i-1 / 2,0\rangle$. This way, a given $z_{i} \in Z_{i}^{M}$ is in the Minkowskian sense above all splitting points $\langle 0, n\rangle \mid n<i$ and above no other splitting points.

Consider now a sequence $Z_{i}$ in $B, Z_{i}=\left\{\left[z_{i} \sigma_{i}\right] \mid i \in \mathbb{N}\right\}$. We will now show that $Z_{i}$ is a chain. Take any $\left[z_{m} \sigma_{m}\right],\left[z_{n} \sigma_{n}\right] \in Z_{i}$ such that $m \neq n$. Either $m<n$ or $n<m$; suppose $m<n$ (the other case is analogous). Since $m<n$, $z_{m} \leqslant \leqslant_{M} z_{n} . z_{m} \in R_{\sigma_{m} \sigma_{n}}$ since it is not above any splitting points between $\sigma_{m}$ and $\sigma_{n}$. Therefore $z_{m} \sigma_{m} \equiv_{S} z_{m} \sigma_{n}$, so $\left[z_{m} \sigma_{m}\right] \leqslant_{S}\left[z_{n} \sigma_{n}\right]$. We have shown that any two elements of $Z_{i}$ are comparable by $\leqslant_{S}$. Therefore, $Z_{i}$ is a chain in $B$, thus being an upward-directed subset of $B$.

The set of all upward-directed subsets of $B$ meets the requirements of the Zorn-Kuratowski Lemma, since a set-theoretical sum of any chain subset of it is also an upward-directed subset of $B$ and is an upper bound of the chain with respect to inclusion. Therefore, there exists a maximal upward-directed subset of $B$ (a history $h^{*}$ ) such that $Z_{i} \subseteq h^{*}$. But lemma 8 is false with respect to this history, since for all $\sigma \in \Sigma, h^{*} \neq\left\{\left[x_{\sigma}\right] \mid x \in \mathbb{R}^{2}\right\}$ ! Suppose 
to the contrary, that for a certain $\sigma \in \Sigma h=\left\{\left[x_{\sigma}\right] \mid x \in \mathbb{R}^{2}\right\}$. As a member of $\Sigma, \sigma$ has to contain a "1" at some point $k$ (starting with 0 ). Then both $\left[z_{k+1} \sigma_{k+1}\right] \in h^{*}$ and $\left[z_{k+1} \sigma\right] \in h^{*}$, so $z_{k+1} \in R_{\sigma_{k} \sigma_{k+1}}$. But $C_{\sigma_{k} \sigma_{k+1}} \ni\langle 0, k\rangle \leqslant_{M}$ $z_{k+1}$, so $z_{k+1} \notin R_{\sigma_{k} \sigma_{k+1}}$ and thus we arrive at a contradiction.

We will now show that our topological postulate [6 is not met in this situation. Consider a chain $Z:=Z_{i}^{M} \cup\{\langle-1,0\rangle\}$. Note that $\langle-1,0\rangle=$ inf $(Z)$. Consider next the chain topology on $\Sigma_{h^{*}}(\langle-1,0\rangle)$ (as defined in the last section) with $Z$ as the original chain. $\left\{\Sigma_{h^{*}}\left(z_{i}\right)\right\}$ is a centred family of closed sets, but its intersection is empty, as $\Sigma$ does not contain a scenario corresponding to the sequence comprised of 0s only. Therefore, we arrived at a contradiction with our corollary 7 , so postulate 6 is not met: the chain topology is not compact.

\section{Conclusions}

Having proved theorem 3, we can adopt Müller's (2002) proof of the fact that $\left\langle B, \leqslant_{S}\right\rangle$ meets all the requirements in definition 2 and conclude that it is a model of BST. We keep in mind, though, that we have introduced a new postulate 6 into the proof and shown that it is not trivial (not always true). We will demand that Minkowskian Branching Structures meet our topological postulate. This way, a MBS is a special kind of BST model: its Our World and ordering $\leqslant$ are constructed as, respectively, $B$ and $\leqslant_{S}$, as proposed by Müller, and furthermore, our postulate 6 is true in the model.

Due to the following self-evident Fact, we have fulfilled our promise from the introduction and produced BST models in which histories are isomorphic to Minkowski space-times.

Fact 10 Let $\mathcal{W}=\left\langle W, \leqslant_{S}\right\rangle$ be an $M B S$ and let $h$ be a history in $\mathrm{W}$ of the form $\left\{\left[x_{\sigma}\right] \mid x \in \mathbb{R}^{4}\right\}$ for a certain $\sigma \in \Sigma$. Then

$$
\left\langle h, \leqslant\left._{S}\right|_{h}\right\rangle \cong\left\langle\mathbb{R}^{4}, \leqslant_{M}\right\rangle
$$

by means of the isomorphism $i: h \rightarrow \mathbb{R}^{4}$ such that $i\left(\left[x_{\sigma}\right]\right)=x$.

Let us observe that in contrast to general BST models, MBS models involve numbers representing spatiotemporal locations, which raises the question of whether the models are frame independent. The best way to read an MBS model $\mathcal{W}$ is to consider it as produced by an idealized omniscient observer associated with a given frame of reference $F$. Of every possible event, the observer knows the event's location (in his/her frame of reference), whether or not the event is a choice point, and what possible continuations the 
event has. There might be another MBS model $\mathcal{W}^{\prime}$ of the same world but produced by another idealized omniscient observer associated with a frame of reference $F^{\prime}$. Since by the assumption, $\mathcal{W}$ and $\mathcal{W}^{\prime}$ represent the same world, there is a one-to-one correspondence $I$ between point events of the two models, with $I\left(\left[x_{\alpha}\right],\left[x_{\alpha^{\prime}}^{\prime}\right]\right)$ read as 'the same event described in two different frames of reference'. We then say that $\mathcal{W}$ and $\mathcal{W}^{\prime}$ are relativistically invariant if for every $\left[x_{\alpha}\right] \in W$ and $\left[x_{\alpha^{\prime}}^{\prime}\right] \in W^{\prime}$ such that $I\left(\left[x_{\alpha}\right],\left[x_{\alpha^{\prime}}^{\prime}\right]\right), x^{\prime}$ is the Lorentz transform of $x$. Thus, a distinction is looming between a single MBS model which is frame-dependent, as it represents the world as seen from some frame of reference, and the relativistically invariant set of MBS models representing the world as seen from all (inertial) frames of reference.

Finally, can we generalize the construction so as to have branching structures produced out of space-times of general relativity? Unfortunately, the answer is no. As already observed in Belnap (1992), in general space-times of general relativity are not upward directed. That is, it is not (generally) true that for incomparable $x, y$ from a given space-time, there is a $z$ such that $x \leqslant z$ and $y \leqslant z$. Yet recall that in BST histories must be upward-directed. Thus, the task of producing generally-relativistic branching structures requires a major overhaul of the BST theory. 


\section{Appendix: splitting points and choice points}

Since it purports to establish that "For histories $h_{\sigma}, h_{\eta} \subset B$ the set $C_{\sigma, \eta}$ is the set of choice points", Lemma 4 in Müller (2002) seems to require reformulation. A splitting point, as a member of $\mathbb{R}^{4}$, is not a member of $B$, and thus is not a choice point.

An obvious move would be to observe that every splitting point $x$ for scenarios $\sigma$ and $\eta$ in a sense "generates" a choice point for histories $h_{\sigma}$ and $h_{\eta}$. That is, if $x \in C_{\sigma \eta}$ then $\left[x_{\sigma}\right]$ is maximal in $h_{\sigma} \cap h_{\eta}$.

What might not be as evident is that, since we have dropped the requirement of finitude of every $C_{\sigma \eta}$, the converse is not true: in some cases there are choice points which are not "generated" in the above way by any splitting points. We will now try to persuade the reader that this is indeed the case. The idea is to use sequences of generated splitting points convergent to the same point. The argument is simple in $\mathbb{R}^{2}$, as we need only two sequences, but it gets more complicated as the number of dimensions increases. (For convenience, in the argument below we use the symbols " $>_{S}$ " and " $>_{M}$ " defined in the natural way and based on " $\leqslant_{S}$ " and " $\leqslant_{M}$ ", respectively.)

Definition 11 1. $S C_{\sigma \eta}:=\left\{\left[c_{\sigma}\right] \mid c \in C_{\sigma \eta}\right\}$

$$
\begin{array}{lll}
2 . \boldsymbol{C}_{\sigma \eta}:=\left\{\left[x_{\gamma}\right]:\right. & \text { (1) }\left[x_{\gamma}\right] \in h_{\sigma} \cap h_{\eta} \text { and } \\
& \text { (2) } \forall z \in \mathbb{R}^{4} \forall \alpha \in \Sigma\left(\left[z_{\alpha}\right]>_{S}\left[x_{\gamma}\right] \Rightarrow\left[z_{\alpha}\right] \notin h_{\sigma} \cap h_{\eta}\right)
\end{array}
$$

" $S C_{\sigma \eta}$ " is to be read as "The set of generated choice points for histories $h_{\sigma}$ and $h_{\eta} "$.

" $\mathrm{C}_{\sigma \eta}$ " is to be read as "The set of choice points for histories $h_{\sigma}$ and $h_{\eta}$ ".

It is of course irrelevant whether we choose $\sigma$ or $\eta$ in square brackets in the definition of the set of generated choice points, since if $c \in C_{\sigma \eta}$ then $c_{\sigma} \equiv_{s} c_{\eta}$ and thus $\left[c_{\sigma}\right]=\left[c_{\eta}\right]$.

Theorem 12 For some $C_{\sigma \eta}, S C_{\sigma \eta} \varsubsetneqq C_{\sigma \eta}$.

Proof sketch. Again, by fixing two spatial dimensions we will restrict ourselves to $\mathbb{R}^{2}$. Let $x=(0,0)$. Let $C_{1}=\{(0,1 / n) \mid n \in \mathbb{N} \backslash\{0\}\}$ and $C_{2}=$ $\{(0,-1 / n) \mid n \in \mathbb{N} \backslash\{0\}\}$. Let $C_{\sigma \eta}=C_{1} \cup C_{2}$. As $x \notin C_{\sigma \eta}$, it is evident that $\left[x_{\sigma}\right] \notin S C_{\sigma \eta}$. We will show that $\left[x_{\sigma}\right] \in \mathbf{C}_{\sigma \eta}$, thus proving the theorem.

We have to show that $\left[x_{\sigma}\right]$ meets conditions (1) and (2) from the above definition. As for (1), $\forall c \in C_{\sigma \eta}$ x SLR c , so $x \in R_{\sigma \eta}$. It follows that $x_{\sigma} \equiv_{S} x_{\eta}$ and finally (as it is obvious that $\left[x_{\sigma}\right] \in h_{\sigma}$ ) that $\left[x_{\sigma}\right] \in h_{\sigma} \cap h_{\eta}$.

Now for (2). Consider $\left[z_{\alpha}\right]$ such that (a) $\left[z_{\alpha}\right]>_{S}\left[x_{\sigma}\right]$. By definition of $>_{S}, z>_{M} x$ and $x_{\alpha}=x_{\sigma}$. Let $z=\left(z_{0}, z_{1}\right)$ (the first coordinate is temporal). 
We distinguish two cases: either the spatial coordinate $z_{1}$ is equal to 0 or it is something else.

If $z=\left(z_{0}, 0\right)$, take $k \in \mathbb{R}, k<z_{0}$ such that $(0, k) \in C_{\sigma \eta}$ (such a $k$ exists since $C_{1}$ converges to $\left.(0,0)\right)$. (*) Since $D_{M}^{2}(z,(0, k))=k-z_{1}<0$, it follows that $x>_{M}(0, k) \in C_{\sigma \eta}$.

On the other hand, if $z_{1} \neq 0$, consider $v$ defined as follows:

$$
v:= \begin{cases}1 & \text { if } z_{1} \geq 1 \\ z_{1} & \text { if } z_{1} \in(0,1) \cup(-1,0) \\ -1 & \text { if } z_{1} \leqslant-1\end{cases}
$$

We choose $(0, k) \in C_{\sigma \eta}$ such that $0<k \leqslant v$ (if $v$ is positive) or $v \leqslant k<0$ (if $v$ is negative). It is always possible to find such a point, since both $C_{1}$ and $C_{2}$ converge to $(0,0)$. We have to prove that (b) $z>_{M}(0, k)$.

From (a) we know that (c) $z>_{M}(0,0)$. To arrive at (b) it suffices to show that (d) $z>_{M}(0, v)$. From (c) it follows that (e) $z_{0} \geq z_{1}$. We have two cases to consider. First, if (f) $z_{1} \geq 1$ or $z_{1} \leqslant-1, D_{M}^{2}(z,(0, v))=-z_{0}^{2}+\left(z_{1}-1\right)^{2}=$ $-z_{0}^{2}+z_{1}^{2}+1-2 z_{1}$, which (by (f) and (e)) is below 0 , which fact is equivalent to (d). Second, if $z_{1} \in(0,1) \cup(-1,0), D_{M}^{2}(z,(0, v))=-z_{0}^{2}+\left(z_{1}-z_{1}\right)^{2}=-z_{0}^{2}$ which is of course negative, so again we arrive at (d).

From $(\mathrm{c})$ and $(\mathrm{d})$ and from the requirement on choosing $(0, k)$ we get the needed result $(\mathrm{b})$.

Since $z>_{M}(0, k) \in C_{\sigma \eta}$, it is true that $z \notin R_{\sigma \eta}$ and thus $\left[z_{\alpha}\right] \notin h_{\sigma} \cap h_{\eta}$. We have thus proved that $\left[x_{\sigma}\right]$ fulfills condition (2).

Unfortunately already in $\mathbb{R}^{3}$ the construction fails at point $\left(^{*}\right)$. To overcome the problem we would have to use four sequences of splitting points convergent to $(0,0,0)$ (intuitively situated at the arms of the coordinate system). To deal with the situation in $\mathbb{R}^{4}$ we would have to similarly introduce six sequences convergent to $(0,0,0,0)$. We will not dwell on the details here, as the point being made does not seem to be significant enough in proportion to the arduous complexity of the argument.

Conjecture 13 For any scenarios $\sigma, \eta \in \Sigma$, the set $\boldsymbol{C}_{\sigma \eta}$ contains exclusively points which belong to $S C_{\sigma \eta}$ or points $\left[x_{\alpha}\right]$ such that $x$ is a limit of a sequence of points belonging to $C_{\sigma \eta}$.

\section{References}

Belnap, N. (1992). Branching space-time. Synthese, 92:385-434. 'Postprint' archived at http://philsci-archive.pitt.edu/archive/00001003. 
Belnap, N. and Szabó, L. (1996). Branching space-time analysis of the GHZ theorem. Foundations of Physics, 26(8):982-1002.

Kowalski, T. and Placek, T. (1999). Outcomes in branching space-time and GHZ-Bell theorems. British Journal for the Philosophy of Science, 50:349375.

Müller, T. (2002). Branching space-time, modal logic and the counterfactual conditional. in T. Placek and J. Butterfield (eds.), Nonlocality and Modality, NATO Science Series, pp. 273-291, Dordrecht. Kluwer Academic Publisher.

Müller, T. (2005). Probability theory and causation: a Branching SpaceTimes analysis. British Journal for the Philosophy of Science, 56(3):487520.

Müller, T. (2006). On the problem of defining the present in special relativity: a challenge for tense logic. in F. Stadler, and M. Stöltzner (eds.), Time and History. Proceedings of the 28. International Ludwig Wittgenstein Symposium, Kirchberg am Wechsel, Austria 2005, pp. 441-458, Frankfurt a.M., Ontos Verlag.

Müller, T. (2007). Branch dependence in the 'consistent histories' approach to quantum mechanics. Foundations of Physics, 37(3):253-276.

Müller, T. and Belnap, N. and Kishida, K. (2006). Funny business in branching space-times: Infinite modal correlations. archived at: http://philsci-archive.pitt.edu/archive/00002803/.

Müller, T. and Placek, T. (2001). Against a minimalist reading of Bell's theorem: Lessons from Fine. Synthese, 128:343-379.

Placek, T. (2004). Quantum state holism: a case for holistic causation. Studies in History and Philosophy of Modern Physics, 35(4):671-692. 